\title{
Case Report \\ Mycoplasma salivarium detected in a microbial community with Candida glabrata in the biofilm of an occluded biliary stent
}

\author{
Correspondence \\ Birgit Henrich \\ birgit.henrich@uni-duesseldorf.de \\ Klaus Pfeffer \\ klaus.pfeffer@uni-duesseldorf.de
}

Received 29 May 2009

Accepted 8 October 2009

\author{
Birgit Henrich, ${ }^{1}$ Marcus Schmitt, ${ }^{2}$ Nora Bergmann,, ${ }^{3}$ Klaus Zanger, ${ }^{4}$ \\ Ralf Kubitz, ${ }^{2}$ Dieter Häussinger ${ }^{2}$ and Klaus Pfeffer $^{1}$ \\ ${ }^{1}$ Institute of Medical Microbiology and Hospital Hygiene, Clinical Centre of Heinrich Heine \\ University, Moorenstrasse 5, 40225 Duesseldorf, Germany \\ ${ }^{2}$ Clinic of Gastroenterology, Hepatology and Infectiology, Clinical Centre of Heinrich Heine \\ University, Moorenstrasse 5, 40225 Duesseldorf, Germany \\ ${ }^{3}$ Centre of Dentistry, Clinical Centre of Heinrich Heine University, Moorenstrasse 5, 40225 \\ Duesseldorf, Germany \\ ${ }^{4}$ Institute of Anatomy II, Clinical Centre of Heinrich Heine University, Moorenstrasse 5, 40225 \\ Duesseldorf, Germany
}

\begin{abstract}
Mycoplasma salivarium, preferentially an inhabitant of the human oral cavity, has rarely been found in other locations associated with disease. We describe here, for what is believed to be the first time, the detection of $M$. salivarium, together with Candida glabrata, in an occluded biliary stent of an icteric, cholestatic patient.
\end{abstract}

\section{Case report}

A 71-year-old man with known alcoholic liver cirrhosis was admitted with painless jaundice, an increase in abdominal circumference, dark urine and acholic faeces of 7 days' duration. Laboratory evaluation indicated abnormal values of C-reactive protein $\left(28 \mathrm{mg} \mathrm{l}^{-1}\right)$, total bilirubin [31.78 mg $\mathrm{dl}^{-1}$ (normal 0.2-1.2)] and direct bilirubin [22.72 $\mathrm{mg} \mathrm{dl}^{-1}$ (normal $<0.30$ )], elevated aspartate aminotransferase (glutamic oxaloacetic transaminase) [113 $\mathrm{U} \mathrm{l}^{-1}$ (normal 0-35)], alanine aminotransferase (glutamic pyruvate transaminase) [107 $\mathrm{U} \mathrm{l}^{-1}$ (normal 0-45)], carcinoembryonic antigen $\left[4.6 \mu \mathrm{g} \mathrm{l}^{-1}\right.$ (normal <3.4)] and carbohydrate antigen [19-9 $448.2 \mathrm{U} \mathrm{ml}^{-1}$ (normal <27)], $\gamma$-glutamyl transferase (standardization according to the IFCC) $\left[104 \mathrm{U} \mathrm{l}^{-1}\right.$ (normal $<55 \mathrm{U}^{-1}$ )], alkaline phosphatase (standardization according to the IFCC) [194 $\mathrm{U} \mathrm{l}^{-1}$ (normal 40-129)].

Abdominal sonography revealed a cirrhotic liver with enlarged intra- and extra-hepatic bile ducts (common bile duct $12 \mathrm{~mm}$ ), gall bladder hydrops, and moderate ascites in the abdomen. Ascites yielded the growth of coagulasenegative staphylococci. The patient was treated with ceftriaxone, and due to hyperthyroidism with carbimazole and perchlorate.

Gastroscopy and endosonography revealed oesophageal varices grade II, ulcera duodeni and portal-hypertensive gastropathy. Furthermore, endosonography and sonogra-

Abbreviations: $\mathrm{BHQ}$, black-hole quencher; $\mathrm{HEX}$, hexachlorofluorescein; IFCC, International Federation of Clinical Chemistry; PE, polyethylene. phy revealed a possible distal cholangio-carcinoma leading to biliary tract obstruction. A malignant stricture was detected in endoscopic retrograde cholangio-pancreotography (ERCP) and in cytology of a brush sample. Drainage of the biliary tract was performed by endoscopic placement of a plastic stent $(60 \mathrm{~mm}, 10$ French diameter $)$ across the stricture.

As bilirubin values remained increased, suggesting early stent occlusion, the stent was replaced by ERCP (thus avoiding contaminations) 2 days later. The main channel and the flaps of the stent were occluded with sludge. Within $24 \mathrm{~h}$ the patient developed temperatures of $39^{\circ} \mathrm{C}$ and became dyspnoeic, respiratory insufficient and acidotic suggestive of early sepsis leading to hypotension and acute renal failure. The patient was transferred to the intensive care unit intubated, and ventilated and continuous venovenous haemofiltration was started.

Bile and blood cultures were positive for Candida glabrata. Despite therapy with voriconazole and imipenem the patient died with clinical signs of sepsis 12 days after admission to hospital.

In the context of studying bacterial communities in biofilm formation, the patient's stent, removed due to occlusion, was used in analysis by PCR. The biofilm was mechanically scraped off the inner surface of the stent. Total DNA was isolated using an EZ1 Biorobot from Qiagen and amplified in fungal 18-28S rDNA PCR with primers $18 \mathrm{~S}-1508 \mathrm{f}\left(5^{\prime}-\right.$ TCC GTA GGT GAA CCT GCG G-3') and 28S-197r (5' TCC TCC GCT TAT TGA TAT GC-3'), and in eubacterial 
$16 \mathrm{~S}$ rDNA PCR with primers $27 \mathrm{f}$ ( $5^{\prime}$-AGA GTT TGA TCN TGG YTC AG-3') and 519r (5'-GWA TTA CCG CRG CKG CTG-3'). Amplification products of the specimen were obtained from both PCRs. Sequencing of the $500 \mathrm{bp}$ 28-18S amplicon followed by a subsequent similarity search using the web-based 'Basic Local Alignment Search Tool' (http://www.ncbi.nlm.nih.gov/BLAST) identified C. glabrata as a fungal pathogen. Analysis of the $16 \mathrm{~S}$ amplicon showed $98 \%$ identity to $16 \mathrm{~S}$ rDNA of Mycoplasma salivarium (accession no. M24661; bp 10499), a cell-wall-less bacterium normally colonizing the oral cavity (Engel \& Kenny, 1970). The presence of $M$. salivarium in the biofilm was confirmed by amplifying an additional region of the 16S rDNA (accession no. M24661; bp 771-1036) using a commercial, mycoplasma-specific PCR (VenorGeM; Minerva Biolabs) and quantified to $10^{7}$ genome equivalents $\mathrm{cm}^{-2}$ stent surface by the use of a rpoB-based TaqMan PCR. This in-house PCR was carried out with the primers Msal-F ( $5^{\prime}$-CCG TCA AAT GAT TTC GAT TGC-3') and Msal-R (5'-GAA CTG CTT GAC GTT GCA TGT T-3') and probe Msal-T (5'-HEX-ATG ATG CTA ACC GTG CGC TTA TGG GTG-BHQ1-3') according to conditions described for Chlamydia trachomatis detection (Schaeffer \& Henrich, 2008). As mycoplasma culture had not been attempted from the stent specimen we verified the ability of $M$. salivarium to survive in bile. Therefore, M. salivarium NCTC 10113 was cultured in arginine medium supplemented with increasing amounts of bile (0 to $100 \%)$ from a cholestatic patient. Bile analysis revealed $0.73 \mathrm{mM}$ taurocholate, $0.65 \mathrm{mM}$ taurochenodeoxycholate, $3.2 \mathrm{mM}$ glycocholate and $3.4 \mathrm{mM}$ glycocheno-deoxycholate. After 2 weeks of cultivation $M$. salivarium had adapted to growth in bile and this strain showed a slightly different protein expression pattern than the original National Collection of Type Cultures strain. By employing scanning electron microscopy we were able to demonstrate colonization of polyethylene (PE) stents with M. salivarium and C. glabrata - even under bile conditions - (Fig. 1a) and micro-colony formation of M. salivarium on PE stents, a prerequisite for biofilm generation (Fig. $1 b)$.

Biliary stenting via endoscopy is the most common palliative treatment for patients suffering from obstructive jaundice associated with malignant hepatobiliary tumours
(Donelli et al., 2007). While in healthy individuals, bile flow and duodenal reflux are under the control of the musculus sphincter of Oddi, which should ensure the sterility of bile and prevent the ascension of microorganisms; loss of the antimicrobial barrier occurs when biliary stents are inserted, especially across the sphincter of Oddi (Sung et al., 1992). As a consequence, biliary stents are covered by a poly-microbial biofilm (Speer et al., 1988) often leading to occlusion by biliary sludge and bacterial biofilms, with the risk of subsequent complications such as hyperbilirubinaemia and sepsis. Knowledge of the colonizing microbial species is not comprehensive but would be useful in facilitating an effective antimicrobial therapy.

Within the last few years an increasing number of microorganisms have been identified in poly-microbial biofilms of biliary stents, with enterococci, Escherichia coli and Klebsiella spp. as the most common aerobic bacteria (Donelli et al., 2007), clostridia as the most common anaerobic bacteria (Leung et al., 2000) and Candida spp. as the most common fungi (Di Rosa et al., 1999).

Although Mollicutes have already been shown to form biofilms (McAuliffe et al., 2006), this is believed to be the first documented case of $M$. salivarium found in a biofilm of an occluded biliary stent. $M$. salivarium preferentially resides as a commensal in the oral cavity of humans (Kundsin \& Praznik, 1967). In some cases it has been considered to participate in oral and peridontal infections (Engel \& Kenny, 1970; Watanabe et al., 1986, 1998). Moreover, M. salivarium was characterized as a causative agent of a submasseteric abscess (Grisold et al., 2008), and of a chronic joint infection in a patient with hypogammaglobulinaemia (So et al., 1983). The presence of M. salivarium DNA in gastric tissue samples of chronic gastritis patients with significant pro-inflammatory responses suggests that this species is not simply a contaminant or facultative-pathogen of the oropharynx (Kwon et al., 2004). A patho-physiological role is supported by the findings that lipoproteins of $M$. salivarium were shown to induce MAPKs p38 and SAPK/JNK, and the transcriptional factors $\mathrm{AP}-1$ and NF- $\kappa \mathrm{B}$ in human fibroblasts (Nakamura et al., 2002), and to induce cell death of lymphocytes and monocytes (Into et al., 2002).

Difficulty in detecting mycoplasmas in routine diagnostic microbiology has complicated the characterization of the (a) PE stent colonized by $M$. salivarium + C. glabrata

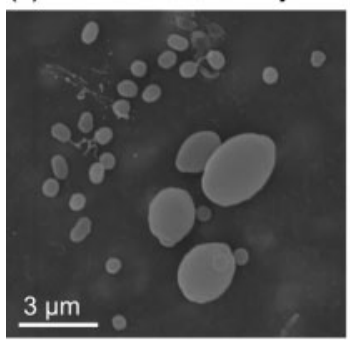

In the presence of bile

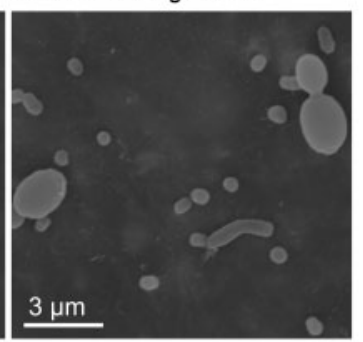

In the absence of bile (b) M. salivarium micro-colony

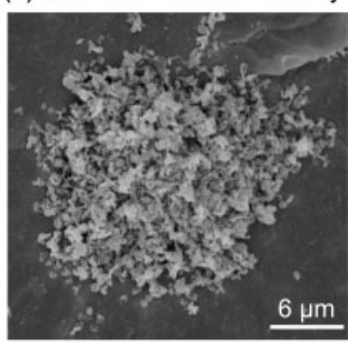

$6 \mu \mathrm{m}$
Fig. 1. Biofilm formation of $M$. salivarium in the presence of bile. (a) Scanning electron microscopy image of $M$. salivarium and $C$. glabrata attached to the PE stent after 5 days of cultivation in arginine medium supplemented with (50\%) or without bile. (b) Scanning electron microscopy image of $M$. salivarium micro-colonies on the PE stent after 20 days of cultivation in arginine medium. 
pathogenicity of these organisms. Cultivation of these small, self replicating, cell-wall-less bacteria with reduced genomes requires complex media and may take days to weeks. In the era of molecular biology methods it has become easier to study bacterial communities independent of culture.

Using PCR, we were able to demonstrate for what is believed to be the first time a Mycoplasma species taking part in biofilm formation of an occluded biliary stent. Stent occlusion is the major complication with biliary stents and occurs in a significant number of cases (10-30\%).

In 1998 Leung and co-workers postulated that there may be a synergistic effect in mixed bacterial infections in biofilm formation on biliary stents (Leung et al., 1998). In our case, routine diagnostics identified C. glabrata as the sole infectious agent. Although anti-mycotic-therapy was started, the patient died with clinical symptoms consistent with Candida sepsis.

The prevalence and pathogenicity of mycoplasmas in the biliary duct and on stents are still unknown, and should, based on this report, be characterized in future work with regard to the bacterial communities they take part in. The recommendation of Diebel et al. (1996) that patients with Candida isolated from biliary stents should be treated with both antifungal and broad-spectrum antimicrobial agents should be considered and possibly reformulated. If mycoplasmas are encountered as pathogens in the biliary duct it should be noted that these cell-wall-less bacteria are resistant to $\beta$-lactam antibiotics, and beside establishing a molecular-biological-based routine diagnostic for mycoplasma, a special antibiotic regime will be required that also includes tetracyclines.

\section{References}

Diebel, L. N., Raafat, A. M., Dulchavsky, S. A. \& Brown, W. J. (1996). Gallbladder and biliary tract candidiasis. Surgery 120, 760-764.

Di Rosa, R., Basoli, A., Donelli, G., Penni, A., Salvatori, F. M., Fiocca, F. \& Baldassarri, L. (1999). A microbiological and morphological study of blocked biliary stents. Microb Ecol Health Dis 11, 84-88.

Donelli, G., Guaglianone, E., Di Rosa, R., Fiocca, F. \& Basoli, A. (2007). Plastic biliary stent occlusion: factors involved and possible preventive approaches. Clin Med Res 5, 53-60.

Engel, L. D. \& Kenny, G. E. (1970). Mycoplasma salivarium in human gingival sulci. J Periodontal Res 5, 163-171.
Grisold, A. J., Hoenigl, M., Leitner, E., Jakse, K., Feierl, G., Raggam, R. B. \& Marth, E. (2008). Submasseteric abscess caused by Mycoplasma salivarium infection. J Clin Microbiol 46, 3860-3862.

Into, T., Okada, K., Inoue, N., Yasuda, M. \& Shibata, K. I. (2002). Extracellular ATP regulates cell death of lymphocytes and monocytes induced by membrane-bound lipoproteins of Mycoplasma fermentans and Mycoplasma salivarium. Microbiol Immunol 46, 667-675.

Kundsin, R. B. \& Praznik, J. (1967). Pharyngeal carriage of mycoplasma species in healthy young adults. Am J Epidemiol 86, 579-583.

Kwon, H. J., Kang, J. O., Cho, S. H., Kang, H. B., Kang, K. A., Kim, J. K., Kang, Y. S., Song, B. C., Kang, H. W. \& other authors (2004). Presence of human mycoplasma DNA in gastric tissue samples from Korean chronic gastritis patients. Cancer Sci 95, 311-315.

Leung, J. W., Liu, Y. L., Desta, T., Libby, E., Inciardi, J. F. \& Lam, K. (1998). Is there a synergistic effect between mixed bacterial infections in biofilm formation on biliary stents? Gastrointest Endosc 48, 250-257.

Leung, J. W., Liu, Y. L., Chan, R. C., Tang, Y., Mina, Y., Cheng, A. F. \& Silva, J., Jr (2000). Early attachment of anaerobic bacteria may play an important role in biliary stent blockage. Gastrointest Endosc 52, 725-729.

McAuliffe, L., Ellis, R. J., Miles, K., Ayling, R. D. \& Nicholas, R. A. (2006). Biofilm formation by mycoplasma species and its role in environmental persistence and survival. Microbiology 152, 913-922.

Nakamura, J., Shibata, K., Hasebe, A., Into, T., Watanabe, T. \& Ohata, N. (2002). Signaling pathways induced by lipoproteins derived from Mycoplasma salivarium and a synthetic lipopeptide (FSL-1) in normal human gingival fibroblasts. Microbiol Immunol 46, 151-158.

Schaeffer, A. \& Henrich, B. (2008). Rapid detection of Chlamydia trachomatis and typing of the lymphogranuloma venereum associated L-serovars by TaqMan PCR. BMC Infect Dis 8, 56.

So, A. K. L., Furr, P. M., Taylor-Robinson, D. \& Webster, A. D. B. (1983). Arthritis caused by Mycoplasma salivarium in hypogammaglobulinaemia. Br Med J 286, 762-763.

Speer, A. G., Cotton, P. B., Rode, J., Seddon, A. M., Neal, C. R., Holton, J. \& Costerton, J. W. (1988). Biliary stent blockage with bacterial biofilm. A light and electron microscopy study. Ann Intern Med 108, 546-553.

Sung, J. Y., Leung, J. W. C., Shaffer, E. A., Lam, K., Olson, M. E. \& Costerton, J. W. (1992). Ascending infection of the biliary tract after surgical sphincterotomy and biliary stenting. J Gastroenterol Hepatol 7, 240-245.

Watanabe, T., Matsuura, M. \& Seto, K. (1986). Enumeration, isolation, and species identification of mycoplasmas in saliva sampled from the normal and pathological human oral cavity and antibody response to an oral mycoplasma (Mycoplasma salivarium). J Clin Microbiol 23, 1034-1038.

Watanabe, T., Shibata, K. I., Yoshikawa, T., Dong, L., Hasebe, A., Domon, H., Kobayashi, T. \& Totsuka, Y. (1998). Detection of Mycoplasma salivarium and Mycoplasma fermentans in synovial fluids of temporomandibular joints of patients with disorders of the joints. FEMS Immunol Med Microbiol 22, 241-246. 